\title{
Potential energy surface of alkali atoms adsorbed on $\mathrm{Cu}(001)$
}

\author{
Guido Fratesi \\ Dipartimento di Scienza dei Materiali and ETSF, Università di Milano-Bicocca, Via Cozzi 53, 20125 Milano, Italy
}

(Received 8 May 2009; revised manuscript received 19 June 2009; published 22 July 2009)

\begin{abstract}
We report a comprehensive density-functional theory (DFT) calculation of the lateral adiabatic potentialenergy surface (PES) of individual alkali atoms adsorbed on the $\mathrm{Cu}(001)$ surface. The corrugation of the PES monotonically decreases by one order of magnitude from Li to $\mathrm{Cs}$, the latter being mobile on the surface even at low temperatures. Substrate relaxations in metastable configurations are consistent with a harmonic response to the adsorbate and account for a significant fraction of the PES corrugation. A simple nonseparable cosine expression provides a good description of values computed between high-symmetry configurations. Results are in good agreement with published He atom scattering measures of surface diffusion, especially at the saddle point, and with structural characterization, pointing out the accuracy of DFT calculations for this class of systems.
\end{abstract}

DOI: $10.1103 /$ PhysRevB.80.045422

PACS number(s): 68.43.Fg, 68.43.Jk, 73.20.-r, 71.15.Mb

\section{INTRODUCTION}

The potential-energy surface (PES) for atoms on the surface of a solid is essential to our understanding of surface phenomena such as diffusion, overlayer growth, and catalysis. In particular, alkali metal adsorbed on metal surfaces have been investigated extensively, both for their relevance in technological applications and for their contribution to the development of fundamental concepts in the theory of adsorption. They have been originally thought as prototypical simple systems within the Langmuir-Gurney model, ${ }^{1,2}$ which has been subsequently challenged and improved by experimental and theoretical studies. ${ }^{3-7}$

Among the experimental techniques providing access to the PES, a remarkable case is given by helium atom scattering (HAS), which enables analyzing the PES from a microscopic point of view, as sampled by adsorbates in diffusion. Alkali atoms on $\mathrm{Cu}(001)$ are systems to which several HAS investigations have been devoted and the understanding of their dynamical properties has proceeded together with improvements to experimental apparata. Typically, experimental findings are coupled to Langevin molecular dynamics (MD) (Ref. 8) (or to Kramer's theory ${ }^{9}$ ) to infer the shape of the PES and extract information on interadsorbate interactions. ${ }^{10}$ Sodium is probably the most studied in the group. The PES of $\mathrm{Na} / \mathrm{Cu}(001)$ at low coverage has been derived from quasielastic HAS since about a decade. ${ }^{11}$ More recently, the much improved resolution of the ${ }^{3} \mathrm{He}$ spin-echo technique $^{12}$ has allowed for a deeper analysis of the diffusion process at larger coverage. ${ }^{13}$ This study interpreted in terms of collective phenomena a coverage dependence of $\mathrm{Na}$ surface diffusion previously reported and unexplained: ${ }^{14,15}$ indeed, the diffusion process involves nontrivial electrondensity rearrangements, ${ }^{16,17}$ a phenomenon further showing the complexity of such systems. Additionally to the information on the given studied system, high-precision HAS measurements may act as a reference to other experimental and theoretical methods.

Theoretical investigations based on first-principles density-functional theory (DFT) simulations have been carried on for decades to elucidate the basic aspects of the ad- sorption of alkali atoms on metals ${ }^{3-5,18}$ as well as to characterize specific atom-substrate combinations. Concerning the determination of the lateral PES, particular attention has been devoted to close-packed substrates since those can exhibit anomalous adsorption sites (i.e., on top instead of hollow $\left.{ }^{19}\right)$. Several other calculations of alkali atoms on metal surfaces rather focus on their interesting surface electronic structure (see, e.g., Ref. 7). Conversely, we are aware of only a few investigations of the lateral PES for alkali metals on $\mathrm{Cu}(001)$. These include studies based on the slab model for the $c(2 \times 2)$ structure $\mathrm{Li} / \mathrm{Cu}(001)$ (Ref. 20); the adsorption of $\mathrm{Na} / \mathrm{Cu}(001)$ at $0.06 \mathrm{ML}$ (Ref. 21); the adsorption of $\mathrm{Cs}$ on $\mathrm{Cu}(001)$ thin films ${ }^{22}$ (it is important to mention that the latter analysis has been performed for an unrealistically large coverage, $0.5 \mathrm{ML}$, about twice as much the saturation one, i.e., $0.27-0.31 \mathrm{ML}$ depending on temperature $\left.{ }^{23,24}\right)$. Up to our knowledge, the only published DFT investigation of the PES for the full alkali-atom group, adsorbed on $\mathrm{Cu}(001)$, is based on small $\mathrm{Cu}_{12}$ clusters, ${ }^{25}$ a limited model as admitted by the author of that study.

In this paper, we shall present a systematic study by $a b$ initio DFT simulations of alkali atoms adsorbed on $\mathrm{Cu}(001)$ at low coverage. We focus on the evaluation of the lateral PES for individual adsorbates. This is a basic ingredient to describe the system at real coverage (to which interadsorbate interactions can then be added ${ }^{26-28}$ ), as well as to describe more complex situations such as coadsorption with other species. Our contribution allows estimation of the PES for the full alkali-atom group and by comparing the results with available experiments, it points out the accuracy of such theoretical methods for this class of systems.

The paper is organized as follows. In Sec. II we describe the theoretical and numerical method, including a procedure to accelerate the convergence of the results with respect to the cell size. In Sec. III we present a short overview of the studied systems and our results for the PES. Section IV discusses the comparison with available experiments, the contribution from substrate deformations, and the PES shape. Finally, Sec. V is devoted to conclusions. 


\section{COMPUTATIONAL METHOD}

The $\mathrm{Cu}(001)$ surface has been modeled by a slab composed by five $\mathrm{Cu}$ layers in a periodically repeated supercells. The size of the supercell in the direction perpendicular to the surface amounts to $12 \mathrm{Cu}(001)$ layers. Alkali atoms were adsorbed on only one side of the metal slab. Dipole-field corrections have been adopted to keep periodicity in the electrostatic potential without spurious electric fields inside the slab, as described by Bengtsson. ${ }^{29}$ The two $\mathrm{Cu}$ layers opposite to the alkali atoms were kept fixed at the bulk-truncated positions while the remaining atoms (including the adsorbate) were relaxed together with the underlying metal layer to minimize the adiabatic total energy with forces smaller than $0.01 \mathrm{eV} / \AA$. All the results are obtained within a generalized gradient approximation (GGA) for the exchangecorrelation functional as proposed by Perdew, Burke, and Ernzerhof (GGA-PBE) ${ }^{30}$ We used the plane-wave ultrasoft pseudopotential method, ${ }^{31}$ as implemented in the PWSCF code of the Quantum-ESPRESSO distribution. ${ }^{32}$ Pseudopotentials were derived from scalar-relativistic all-electron atomic calculations. Semicore $s p$ states from the alkali atoms (with the exception of $\mathrm{Li}$ ) are included in the valence together with $3 d 4 s \mathrm{Cu}$ ones. Wave functions are expanded up to a kineticenergy cutoff of 27-42 Ryd to ensure convergency; the effective potential and the charge density up to 216 Ryd. The surface Brillouin-zone integration has been performed with the Monkhorst-Pack ${ }^{33}$ scheme, adopting $\mathbf{k}_{\|}$-point meshes equivalent to a $12 \times 12$ mesh in the irreducible surface unit cell of $\mathrm{Cu}(001)$. The calculated nearest-neighbor spacing in $\mathrm{Cu}$ is $2 r_{\mathrm{Cu}}=2.57 \AA$ [experimental: $2.55 \AA$ (Ref. 34)]. Tests were performed to ensure convergency of the results with respect to this numerical setup, for example, by varying the number of $\mathrm{Cu}$ layers in the slab (see also the dependence of $\mathrm{Na} / \mathrm{Cu}(001)$ energy on the number of layers reported in Ref. 21). The main results of this work would also be obtained (PES corrugation within $5-10 \mathrm{meV}$ ) by using less accurate pseudopotentials without semicore states; however, this would affect significantly the computed dipole moments.

The adsorption energy per adatom $\mathrm{A}$ is then computed as

$$
E^{\mathrm{ads}}=E_{\mathrm{A} / \text { surf }}^{\mathrm{tot}}-E_{\mathrm{surf}}^{\mathrm{tot}}-E_{\mathrm{A}}^{\mathrm{tot}}
$$

where the terms on the right-hand side are the total energy of the atom A adsorbed on the surface, of the clean surface, and of the free neutral atom computed in a suitably large supercell.

In Eq. (1), the adatom coordinates parallel to the surface $(x, y)$ can be fixed while relaxing the one perpendicular to the surface $(z)$ and surface-atom degrees of freedom. Hence, a two-dimensional adiabatic PES for the alkali adatom is obtained, with periodicity of the $\mathrm{Cu}(001)$ surface [shown in Fig. 1(a)]. By taking the hollow $(H)$ site as energy reference and origin for $(x, y)$ coordinates, a simple model for the PES is given by including only the first Fourier components along $\langle 100\rangle$ and $\langle 110\rangle$ directions, as proposed, for example, in Ref. 15 , (a)

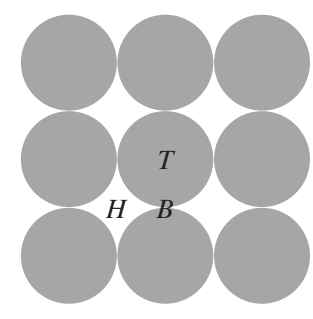

(b)

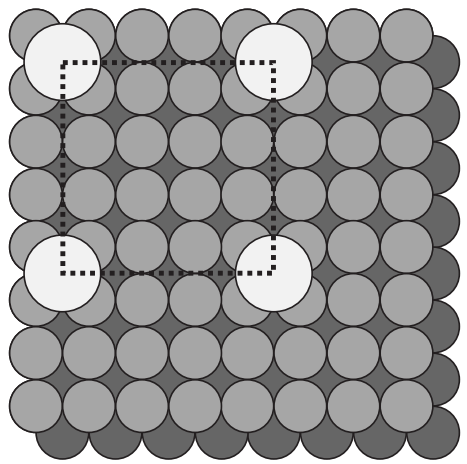

FIG. 1. (a) Schematic top view of the $\mathrm{Cu}(001)$ surface indicating the high-symmetry hollow $(H)$, bridge $(B)$, and top $(T)$ adsorption sites. (b) Top view of the $(4 \times 4)$ unit cell adopted in the calculations (indicated by the dashed line). Bright and dark circles mark alkali and $\mathrm{Cu}$ atoms, respectively.

$$
\begin{aligned}
V(x, y) \equiv & E^{\mathrm{ads}}(x, y)-E_{H}^{\mathrm{ads}} \\
= & \frac{E_{T}}{4}+\frac{E_{B}}{2}-\frac{E_{T}}{4}\left[\cos \left(\frac{2 \pi x}{a}\right)+\cos \left(\frac{2 \pi y}{a}\right)\right] \\
& +\frac{E_{T}-2 E_{B}}{4} \cos \left(\frac{2 \pi x}{a}\right) \cos \left(\frac{2 \pi y}{a}\right)
\end{aligned}
$$

The above expression only depends on the values of the relative energies in bridge $(B)$ and top $(T)$ sites, $E_{T}$ and $E_{B}$, respectively, which can be varied independently; $a$ is the $\mathrm{Cu}(001)$ surface lattice constant (i.e., the distance between adjacent $H$ sites, $\left.a=2 r_{\mathrm{Cu}}\right)$. Notice that the second and third terms on the right-hand side of Eq. (1) are common to all adsorption sites and cancel in determining $V$.

To evaluate the PES for adsorbates in the low-coverage regime by finite-size supercell calculations, one should take the limit for infinite cell size since the adsorption energy and its dependence on $(x, y)$ vary as a function of the simulation cell. This is shown in Fig. 2, where the adsorption energy in the hollow site and the relative $B$ and $T$ energies are reported as a function of the simulation cell (filled symbols) for a calculation of $\mathrm{Na} / \mathrm{Cu}(001)$. Rectangular $(n \times m)$ unit cells with $n, m=3 a, 4 a$, and $6 a$ have been used, adopting here for numerical convenience a less refined model than earlier specified (three $\mathrm{Cu}$ layers in the slab and unrelaxed geometries). Equivalent $k$-point meshes in the Brillouin-zone integration make the results directly comparable. For reasons which will now become evident, the results are shown as a function of the quantity $S$,

$$
S=\sum_{j \neq 0} \frac{1}{4 \pi \varepsilon_{0}} \frac{1}{\left|\mathbf{R}_{j}-\mathbf{R}_{0}\right|^{3}}
$$

( $\mathbf{R}_{j}$ being the surface coordinate of the $j$ th periodic replica of the adatom in $\mathbf{R}_{0}$ ), which scales as $\Theta^{3 / 2}$ and allows one to compare on the same footing the outcome of different cell shapes. A fit through the data (solid lines) and the extrapolation to $S \rightarrow 0$ provides the wanted limit, where the adatom and its replica are infinitely far from each other. Notice that the energy differences $E_{B}$ and $E_{T}$ (middle and bottom panels) are less affected by the finite size of the unit cell than $E^{\text {ads }}$ 


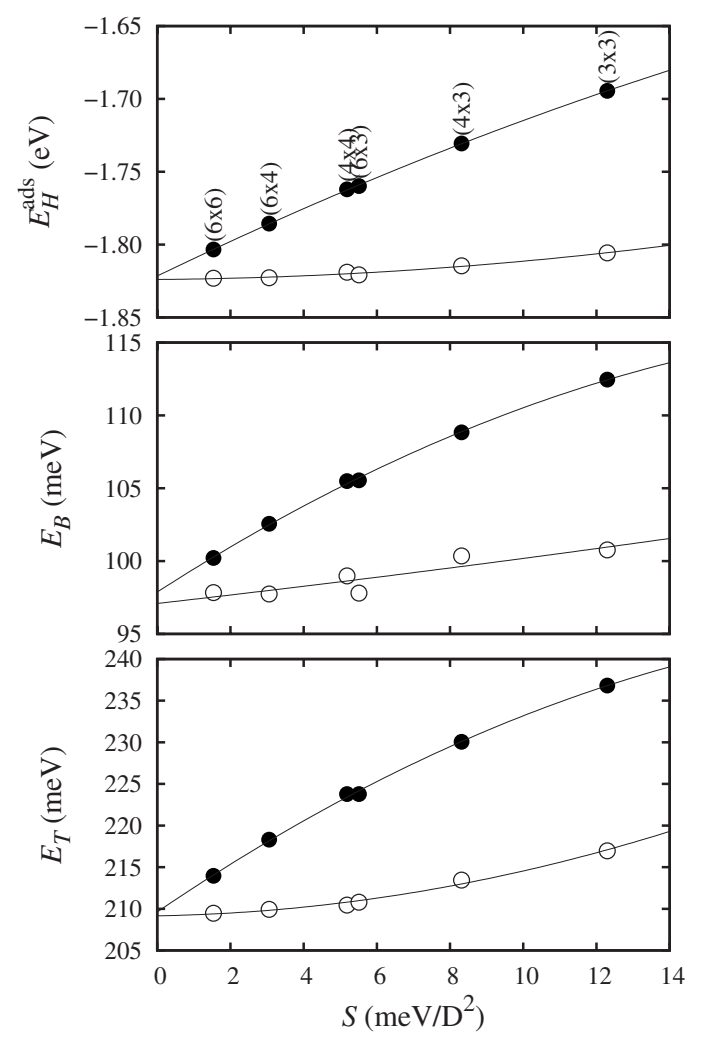

FIG. 2. Example of extracting the limit to isolated adatoms for a simplified model of $\mathrm{Na} / \mathrm{Cu}(001)$ chosen for numerical convenience (details in the text). Adsorption energy in hollow ( $E_{H}^{\text {ads }}$, top panel), bridge-hollow and top-hollow energy differences $\left(E_{B}\right.$ and $E_{T}$, middle and bottom panels) are reported for different $(n \times m)$ unit cells as a function of the cell-dependent quantity $S$ [Eq. (3)]. Filled circles: bare result; empty symbols: upon removal of the dipoledipole interaction energy, see Eqs. (5) and (6). Solid lines are quadratic fits to the points.

but still for the $(4 \times 4)$ unit cell they are at about $10 \%$ from the converged value.

A much simpler procedure is suggested by the good linearity of the data shown in Fig. 2, which points out that the major contribution to the interaction of the adsorbate with its replicas is of dipole-dipole origin. Indeed, an arrangement of ideal electric dipoles on a metal surface has the energy (per dipole)

$$
E^{\mathrm{dd}}=\frac{1}{2} \sum_{j \neq 0} \frac{1}{4 \pi \varepsilon_{0}} \frac{2 \mu^{2}}{\left|\mathbf{R}_{j}-\mathbf{R}_{0}\right|^{3}}=\mu^{2} S .
$$

Here a factor 2 taking into account the image dipole cancels a factor $1 / 2$ for the summation over the couples. The value of $E^{\mathrm{dd}}$ has been previously adopted as a measure of local concentration of adsorbates for generic distributions of alkali atoms. ${ }^{13,16}$ As already done for $\mathrm{Na},{ }^{21}$ we define a refined adsorption energy by subtracting such dipole-dipole contribution, using the value of $\mu$ obtained by the corresponding DFT calculation

$$
\bar{E}^{\mathrm{ads}}=E^{\mathrm{ads}}-E^{\mathrm{dd}} .
$$

Similarly, we define the relative energy $\bar{E}_{B}$ (and analogously, $\bar{E}_{T}$ ) by taking as reference the refined adsorption energy in the hollow site, $\bar{E}_{H}^{\text {ads }}$ as

$$
\bar{E}_{B} \equiv \bar{E}_{B}^{\mathrm{ads}}-\bar{E}_{H}^{\mathrm{ads}}=E_{B}-E_{B}^{\mathrm{dd}}+E_{H}^{\mathrm{dd}} .
$$

Notice that the dipole-dipole contribution to the PES corrugation does not cancel because the dipole moment (and consequently the spurious interaction among the replicas) is a function of the adsorption configuration. The refined quantities $\bar{E}_{H}^{\text {ads }}, \bar{E}_{B}$, and $\bar{E}_{B}$ are also reported in Fig. 2 as empty symbols. Their limits for $S \rightarrow 0$ are the same as the ones for $E^{\text {ads }}, E_{B}$, and $E_{T}$. Remarkably, they are much more independent of the simulation cell than the corresponding uncorrected values, in particular, their values computed with the $(4 \times 4)$ unit cell are already very close to the result extrapolated for $S \rightarrow 0$. These reasons allow us to estimate the limit of the PES for infinitely large supercell simply by use of Eq. (6) and a $(4 \times 4)$ simulation cell, shown in Fig. 1(b).

\section{RESULTS}

It is well known that the adsorption of alkali atoms induces a significant charge rearrangement in the region around the adatom. Before presenting our results on the adiabatic PES in detail, let us overview this important issue, referring to the most stable hollow site, at $\Theta=1 / 16 \mathrm{ML}$. In Fig. 3 we show the difference between the electron density of the adatom/surface system and the sum of those of the neutral surface and adatom fragments. Left and right panels refer to (010) and (1 $\overline{1} 0)$ planes, respectively, passing through the adatom. Such charge displacement is associated with a large dipole moment, $\mu$, and a corresponding reduction in the surface work function, $\Delta \Phi$. Our results, reported in Table I, show the larger $\Delta \Phi$ for the heavier alkalis to be in good agreement with experimental values. Qualitative features are similar for all adatoms. Notice in Fig. 3 a polarization of the region around the adatom, with electron density transferred from above it to the region in between the alkali atom and the surface. Heavier alkalis induce a less intense, yet broader in space, electron-density rearrangement, resulting in an overall larger dipole moment; an opposite polarization originating in the semicore $s p$ shell is also apparent, which reduces the total $\mu$ (as an example, by neglecting semicore $s p$ states in the pseudopotential, the dipole moment of adsorbed Cs would be $11 \%$ larger).

We now discuss the structures obtained by adsorbing the adatom in $H, B$, and $T$ sites. Those are schematically depicted in Fig. 4 and the main structural parameters are collected in Table II. We remind that the surface $(x, y)$ coordinates of the adatom are kept frozen and all other coordinates (including the adatom height $z$ ) are optimized.

In the hollow site, the adatom distance from the surface, $z_{\mathrm{A}}$, is in the range 1.9-3.0 $\AA$, the higher value for the heavier atom. Corresponding values of the bond length, $d_{\mathrm{A}-\mathrm{Cu}}$, and of the effective radius $r_{\mathrm{A}}=d_{\mathrm{A}-\mathrm{Cu}}-r_{\mathrm{Cu}}$, are also re- 

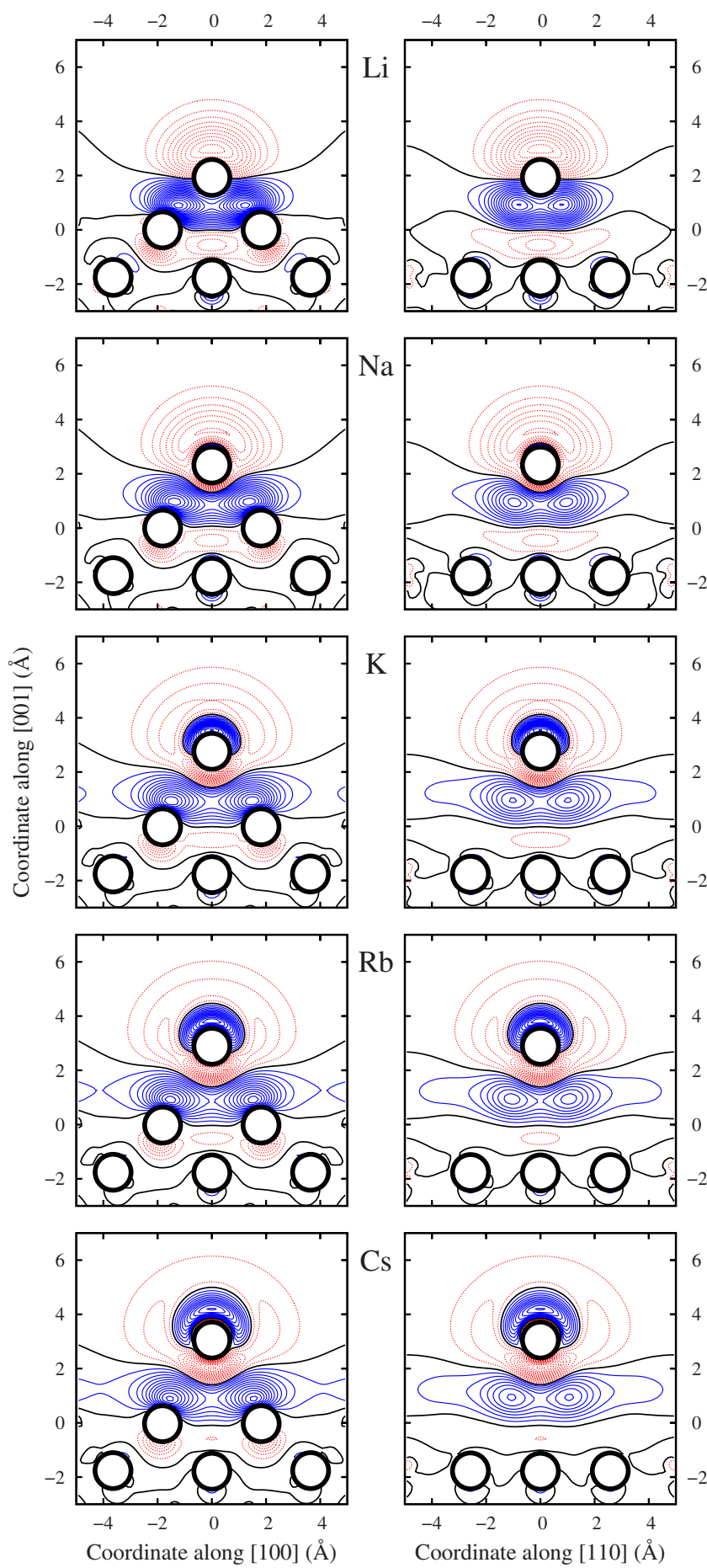

FIG. 3. (Color online) Charge displacement upon adsorption, with respect to the density of the clean surface and the neutral free atom. Left panels report the results for Li to Cs (from top to bottom) on a (010) plane cutting the adatom; right panels refer instead to a (11̄0) plane. Isolines are shown every $0.002 \AA^{-3}$. Dotted (red), thick solid (black), and thin solid (blue) lines refer to positive, zero, and negative charge displacement. Circles mark the position of atoms in the plane.

ported in Table II. Those are systematically larger than the ionic ones $\left(r_{\mathrm{A}}^{\mathrm{ion}}\right)$. One notices that the difference $r_{\mathrm{A}}-r_{\mathrm{A}}^{\text {ion }}$ $=0.62 \pm 0.02 \mathrm{~A}$ is constant across the series, similarly to the observation recently made in Ref. 37 for the adsorption height of alkali atoms on $\mathrm{Cu}(111)$. Experimental studies yield $r_{\mathrm{Li}}=1.39 \pm 0.08 \AA{ }^{38} r_{\mathrm{Na}}=1.70 \AA,{ }^{39} r_{\mathrm{K}}=1.61 \AA,{ }^{40}$ and $r_{\mathrm{Cs}}=2.18 \AA .^{40}$ Values quoted here refer to different coverages than the $(4 \times 4)$ of our investigation but we remember that the bond length for adsorbed alkali atoms is only mildly dependent on coverage. ${ }^{6}$ While our values are generally in very good agreement with these experimental findings (as also achieved by DFT-GGA calculations for alkali-metalnoble-metal diatomic molecules ${ }^{41}$ ), the value quoted for $\mathrm{K}$ in Ref. 40 is significantly lower than our result $\left(r_{K}=2.01 \AA\right)$. Notice that this experimental value is surprisingly smaller than the one for $\mathrm{Na}^{39}$ Other structural studies for $\mathrm{K}$ adsorbed on metals in surface sites give an effective radius in the range 1.6-2.0 $\AA$ and 1.5-1.8 $\AA$ for $\mathrm{Na}^{42}$ Other DFT calculations, based on the local-density approximation (LDA), reported slightly smaller values than the ones reported here (as generally expected when comparing PBE and LDA results): $r_{\mathrm{A}}=1.3,1.5$, and $1.8 \AA$ for $\mathrm{A}=\mathrm{Li}, \mathrm{Na}$, and $\mathrm{K} .{ }^{20,43}$ Notice again a larger value for $\mathrm{K}$ than in Ref. 40 while $\mathrm{Li}$ and $\mathrm{Na}$ results are smaller than experimental values quoted above.

The height of adsorption $z_{\mathrm{A}}$ increases when the adatom is at the bridge or top position, especially for the smaller alkalis. Such variation is lower than that obtained in case of constant $\mathrm{A}-\mathrm{Cu}$ bond length (i.e., the effective radius lowers in $B$ and $T$ ). As for substrate deformations induced by the adsorbate, only small atomic displacement, with respect to the coordinates of the clean $\mathrm{Cu}(001)$ surface, are induced when the adsorbate is in the hollow site: the norm of the overall displacement vector, $\|\Delta \mathbf{R}\|$, is lower than $0.1 \AA$ for all alkali atoms. In bridge and top sites larger deformations are found. In particular, the $\mathrm{Cu}$ atoms adjacent to the alkali one are pushed inward by the quantity $\Delta z_{\mathrm{Cu}}$; in the plane, they are displaced farther from the alkali atom by the smaller quantity $\Delta x_{\mathrm{Cu}}$. See Fig. 4 and Table II. The position of the other atoms in the surface layer is not much affected by the presence of the adsorbate. Among the second layer, the largest displacement is the one of the $\mathrm{Cu}$ atoms closer to the adatom (one $\mathrm{Cu}$ atom when the alkali is in $H$, two in $B$, and four in $T$ sites), indicated as $\Delta z_{\mathrm{Cu}}^{(2)}$, which is directed inward. No displacement larger than $0.01 \AA$ has been found among the $\mathrm{Cu}$ atoms in the third layer.

Finally, we report in Table III our estimate of the lateral PES parameters, $E_{B}$ and $E_{T}$ [see Eq. (2)], together with the corresponding values, $\bar{E}_{B}$ and $\bar{E}_{T}$, corrected for the dipoledipole interaction with the periodic replicas in the calculation by use of Eq. (6). As already found in the model calculation discussed in Sec. II, neglecting this correction results in slightly overestimating the corrugation of the PES. For all atoms, the hollow site is the most stable one, bridge is a saddle point of $V(x, y)$, and top is a maximum. The energy in top is slightly smaller than twice the one in bridge (on the average, $E_{T} / E_{B}=1.8$ ). The corrugation of the PES for alkali atoms on $\mathrm{Cu}(001)$ decreases significantly from $\mathrm{Li}$ to $\mathrm{Cs}$, implying that the latter atom will be much more mobile. For $\mathrm{Li}$, the values for $E_{B}$ and $E_{T}$ are relatively large, considering that at room temperature $\mathrm{Li}$ induces reconstructions of the $\mathrm{Cu}(001)$ surface (missing row, with alkali atoms along the 
TABLE I. Dipole moment, $\mu,(D)$ and work-function reduction, $\Delta \Phi,(\mathrm{eV})$. Experimental values are from Refs. 35, 36, and 23. They have been measured at a coverage of $0.06 \mathrm{ML}$, with the exception of Na $(0.07$ ML).

\begin{tabular}{lccccc}
\hline \hline & $\mathrm{Li}$ & $\mathrm{Na}$ & $\mathrm{K}$ & $\mathrm{Rb}$ & $\mathrm{Cs}$ \\
\hline$\mu$ & 2.02 & 3.11 & 4.78 & 5.20 & 5.53 \\
$\Delta \Phi$ & 0.72 & 1.11 & 1.70 & 1.85 & 1.97 \\
$\Delta \Phi^{\exp }$ & $0.7^{\mathrm{a}}$ & $1.4^{\mathrm{a}}$ & $1.8^{\mathrm{b}}$ & & $2.0^{\mathrm{c}}$ \\
\hline \hline
\end{tabular}

Reference 35 .

${ }^{\mathrm{b}}$ Reference 36 .

${ }^{\mathrm{c}}$ Reference 23.

troughs, already at a coverage of $0.25 \mathrm{ML}{ }^{45}$ As for Cs adatoms, their diffusion would be dominated by the strong repulsive interaction with the neighbors. ${ }^{46}$

\section{DISCUSSION}

Our value for the saddle-point energy for $\mathrm{Na} / \mathrm{Cu}(001)$ is in excellent agreement with the existing HAS-MD estimates: ${ }^{11}$ we obtain $79 \mathrm{meV}$, to be compared with $75 \mathrm{meV}$. A larger deviation is found for the energy on top, for which our DFT value, $143 \mathrm{meV}$, is larger than the HAS-MD one, $84 \mathrm{meV}$. A very similar DFT estimate of the PES corrugation in the top site is obtained by an LDA calculation $\left(\bar{E}_{B}=78 \mathrm{meV}\right.$ and $\bar{E}_{T}=145 \mathrm{meV}$ ). Preliminary results by HAS are also available for $\mathrm{K} / \mathrm{Cu}(001)$, showing a good agreement of MD simulations based on our results with experimental data, both for bridge and top energies. ${ }^{47}$ As for HAS experiments on Cs/ $\mathrm{Cu}(001)$, owing to low experimental amplitude at large momentum transfer, it has not been possible to determine the top/bridge energy ratio; therefore, a substrate potential with a single parameter, $E_{T} \equiv E_{B}=20 \pm 2 \mathrm{meV}$, has been proposed. ${ }^{46}$ This value is intermediate between our estimates for the bridge and top energies, 13 and $25 \mathrm{meV}$, respectively. One has to notice that the PES corrugation for alkalis on $\mathrm{Cu}(001)$, on the order of a few tenths of $\mathrm{eV}$, is about the same magnitude as the chemical accuracy searched and not commonly achieved by exchange-correlation functionals such as PBE. Nevertheless, error compensations apparently occur among adsorption in various surface sites, which share similar overall properties. The case of $\mathrm{Na}$ shows that such compensation is more effective between $B$ and $H$, rather than $T$ and $H$ sites. (a)

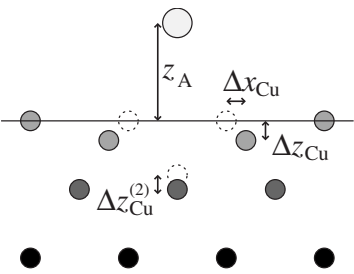

(b)

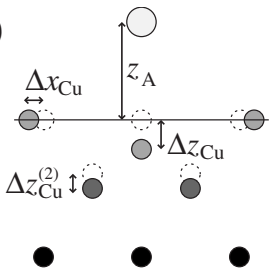

FIG. 4. Schematic side view of the adsorption structure defining the parameters tabulated in Table II for (a) hollow and bridge and (b) top sites. The solid line marks the position of the first $\mathrm{Cu}$ layer for the clean surface (chosen as a reference) while dashed circles the unrelaxed coordinates of $\mathrm{Cu}$ atoms nearest to the alkali.
Other published DFT calculations of the PES of alkali atoms on $\mathrm{Cu}(001)$ have been performed, up to our knowledge, only at much larger coverage. For the $\mathrm{Cu}(001)-c(2$ $\times 2$ )-Li the hollow site is predicted to be more stable than the top one by $355 \mathrm{meV}^{20}$ For the hypothetical $\mathrm{Cu}(001)-c(2 \times 2)$-Cs (with coverage much larger than the saturation one, ${ }^{23,24}$ as mentioned in Sec. I), top and bridge are both higher in energy than the hollow site by about 15 meV. ${ }^{22}$ Finally, energy differences are too small for the results of the $\mathrm{Cu}_{12}$ cluster investigation reported in Ref. 25 to be reliable, concerning the PES; top site adsorption would be even predicted to be more stable for $\mathrm{K}, \mathrm{Rb}$, and $\mathrm{Cs}$.

A significant fraction of the PES corrugation originates in contributions from the substrate deformations induced by the adsorption described in Sec. III. To see this, we report in Table IV the energy spent in deforming the substrate from the relaxed configuration of the clean surface (which has total energy $\left.E_{\text {surf }}^{\text {tot }}\right)$ to the geometry induced by the adsorbate (with total energy $E_{\text {surf-def }}^{\text {tot }}$ ),

$$
E^{\text {def }}=E_{\text {surf-def }}^{\text {tot }}-E_{\text {surf }}^{\text {tot }}>0 .
$$

The contribution of this energy cost to the PES corrugation in bridge and top sites is then given by the differences $E_{B}^{\text {def }}$ $-E_{H}^{\mathrm{def}}$ and $E_{T}^{\mathrm{def}}-E_{H}^{\mathrm{def}}$, respectively. It evaluates up to $70 \mathrm{meV}$ for $\mathrm{Li}$ in the top site, although it becomes more important

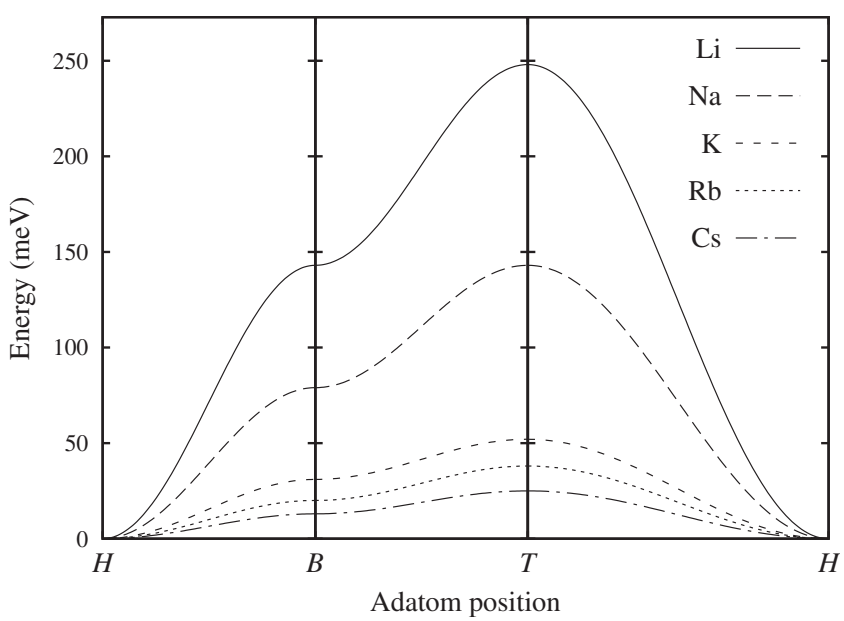

FIG. 5. Potential-energy surface, $V(x, y)$, between highsymmetry adsorption sites. 
TABLE II. Structural parameters $(\AA): z_{\mathrm{A}}$, adatom adsorption height; $d_{\mathrm{A}-\mathrm{Cu}}$, bond length; $r_{\mathrm{A}}$, effective radius; $\Delta x_{\mathrm{Cu}}$ and $\Delta z_{\mathrm{Cu}}$, lateral and perpendicular displacement of $\mathrm{Cu}$ atoms nearest to the alkali atom; $\Delta z_{\mathrm{Cu}}^{(2)}$, perpendicular displacement of atoms in the second layer; $\|\Delta \mathbf{R}\|$, norm of the displacement vector for $\mathrm{Cu}$ atoms with respect to a clean surface. See also Fig. 4. Ionic radii, $r_{\mathrm{A}}^{\text {ion }}$, are given for octahedral coordination (Ref. 44).

\begin{tabular}{lccccc}
\hline \hline & $\mathrm{Li}$ & $\mathrm{Na}$ & $\mathrm{K}$ & $\mathrm{Rb}$ & $\mathrm{Cs}$ \\
\hline$r_{\mathrm{A}}^{\text {ion }}$ & 0.76 & 1.02 & 1.38 & 1.52 & 1.67
\end{tabular}

Hollow

\begin{tabular}{|c|c|c|c|c|c|}
\hline$z_{\mathrm{A}}$ & 1.93 & 2.33 & 2.73 & 2.89 & 3.02 \\
\hline$d_{\mathrm{A}-\mathrm{Cu}}$ & 2.65 & 2.95 & 3.30 & 3.43 & 3.55 \\
\hline$r_{\mathrm{A}}$ & 1.37 & 1.67 & 2.01 & 2.14 & 2.27 \\
\hline$\Delta x_{\mathrm{Cu}}$ & 0.00 & 0.00 & 0.00 & 0.00 & 0.00 \\
\hline$\Delta z_{\mathrm{Cu}}$ & 0.00 & 0.00 & 0.02 & 0.02 & 0.03 \\
\hline$\Delta z_{\mathrm{Cu}}^{(2)}$ & 0.02 & 0.02 & 0.03 & 0.03 & 0.03 \\
\hline$\|\Delta \mathbf{R}\|$ & 0.06 & 0.06 & 0.07 & 0.08 & 0.09 \\
\hline \multicolumn{6}{|c|}{ Bridge } \\
\hline$z_{\mathrm{A}}$ & 2.07 & 2.40 & 2.75 & 2.92 & 3.03 \\
\hline$d_{\mathrm{A}-\mathrm{Cu}}$ & 2.49 & 2.78 & 3.10 & 3.24 & 3.34 \\
\hline$r_{\mathrm{A}}$ & 1.21 & 1.49 & 1.81 & 1.95 & 2.06 \\
\hline$\Delta x_{\mathrm{Cu}}$ & 0.01 & 0.01 & 0.01 & 0.00 & 0.01 \\
\hline$\Delta z_{\mathrm{Cu}}$ & 0.06 & 0.05 & 0.07 & 0.05 & 0.06 \\
\hline$\Delta z_{\mathrm{Cu}}^{(2)}$ & 0.04 & 0.03 & 0.03 & 0.03 & 0.03 \\
\hline$\|\Delta \mathbf{R}\|$ & 0.15 & 0.13 & 0.13 & 0.11 & 0.12 \\
\hline \multicolumn{6}{|l|}{ Top } \\
\hline$z_{\mathrm{A}}$ & 2.16 & 2.47 & 2.81 & 2.93 & 3.03 \\
\hline$d_{\mathrm{A}-\mathrm{Cu}}$ & 2.33 & 2.60 & 2.93 & 3.04 & 3.14 \\
\hline$r_{\mathrm{A}}$ & 1.04 & 1.32 & 1.64 & 1.75 & 1.85 \\
\hline$\Delta x_{\mathrm{Cu}}$ & 0.04 & 0.03 & 0.02 & 0.02 & 0.01 \\
\hline$\Delta z_{\mathrm{Cu}}$ & 0.17 & 0.14 & 0.12 & 0.11 & 0.10 \\
\hline$\Delta z_{\mathrm{Cu}}^{(2)}$ & 0.05 & 0.04 & 0.04 & 0.03 & 0.03 \\
\hline$\|\Delta \mathbf{R}\|$ & 0.25 & 0.19 & 0.17 & 0.15 & 0.14 \\
\hline
\end{tabular}

relatively to $V$ for the heavier alkalis (it amounts to about $50 \%$ the PES corrugation in Cs).

It is also interesting to notice that such cost to deform the substrate to the adsorption configuration is very close to the energy gained by allowing substrate deformations in adsorption, i.e., when total energies of the adatom adsorbed on a surface with $\mathrm{Cu}$ coordinates frozen $\left(E_{\mathrm{A} / \text { surf-frozen }}^{\text {tot }}\right)$ or allowed to relax $\left(E_{\mathrm{A} / \text { surf }}^{\text {tot }}\right)$ are compared,

$$
E^{\text {relax }}=E_{\mathrm{A} / \text { surf-frozen }}^{\mathrm{tot}}-E_{\mathrm{A} / \text { surf }}^{\mathrm{tot}}>0 .
$$

See again Table IV for the values. We found that the average of $\left|E^{\text {def }}-E^{\text {relax }}\right|$ is only $2 \mathrm{meV}$. The equality $E^{\text {relax }}=E^{\text {def }}$ follows analytically if one assumes that the adsorbate induces a perturbation, linearly depending on atomic coordinates, on a substrate whose response is harmonic, and breaks if higherorder terms are included. ${ }^{48}$

TABLE III. Parameters for the PES model in Eq. (2) $(\mathrm{meV})$, computed in a $(4 \times 4)$ unit cell $\left(E_{B}\right.$ and $\left.E_{T}\right)$ and with the correction for the interaction with periodic replicas by use of Eq. (6) $\left(\bar{E}_{B}\right.$ and $\left.\bar{E}_{T}\right)$.

\begin{tabular}{lccccc}
\hline \hline & $\mathrm{Li}$ & $\mathrm{Na}$ & $\mathrm{K}$ & $\mathrm{Rb}$ & $\mathrm{Cs}$ \\
\hline$E_{B}$ & 152 & 86 & 35 & 24 & 16 \\
$E_{T}$ & 264 & 156 & 64 & 45 & 30 \\
$\bar{E}_{B}$ & 143 & 79 & 31 & 20 & 13 \\
$\bar{E}_{T}$ & 248 & 143 & 52 & 38 & 25 \\
\hline \hline
\end{tabular}


TABLE IV. Substrate-deformation contributions to the adsorption energy: $E^{\text {def }}$, energy cost for the substrate deformation induced by the adsorbate [Eq. (7)]; $E^{\text {relax }}$, energy gain to relax the substrate coordinates in the compound system [Eq. (8)]. Values in meV.

\begin{tabular}{lccccc}
\hline \hline & $\mathrm{Li}$ & $\mathrm{Na}$ & $\mathrm{K}$ & $\mathrm{Rb}$ & $\mathrm{Cs}$ \\
\hline$E_{H}^{\text {def }}$ & 7 & 5 & 6 & 6 & 10 \\
$E_{B}^{\text {def }}$ & 31 & 22 & 20 & 15 & 17 \\
$E_{T}^{\text {def }}$ & 76 & 48 & 32 & 25 & 24 \\
$E_{H}^{\text {relax }}$ & 4 & 5 & 7 & 7 & 8 \\
$E_{B}^{\text {relax }}$ & 25 & 21 & 19 & 17 & 17 \\
$E_{T}^{\text {relax }}$ & 70 & 50 & 34 & 31 & 26 \\
\hline \hline
\end{tabular}

Another interesting result of this study is that the simple formula in Eq. (2), given to interpolate the PES at highsymmetry adsorption sites, is actually an excellent approximation to the DFT results for all adatom positions. This was assessed by performing structural relaxations with the adatom $(x, y)$ coordinates fixed at the midpoints between $H, B$, and $T$. Such a choice is equivalent to a $4 \times 4$ sampling of the surface unit cell and would allow determining the next components in the Fourier expansion of $V$. Resulting energies differ by no more than $3 \mathrm{meV}$ from those given by Eq. (2). The same conclusion was further validated by finer scans of the PES along the [100], [110], and [010] directions for $\mathrm{Na}$, $\mathrm{K}$, and $\mathrm{Cs}$, and by a $16 \times 16$ sampling of the surface unit cell for $\mathrm{K}$.

The PES given by these DFT simulations has therefore a simple sinusoidal shape along the [100], [110], and [010] directions connecting the high-symmetry adsorption sites, as described by Eq. (2) and shown in Fig. 5 (further notice, in the same figure, the larger mobility of the heavier alkali atoms). It is worth mentioning that a more complicated expression for the PES of $\mathrm{Na} / \mathrm{Cu}(001)$ has been proposed as a best fit to single atom MD simulations. ${ }^{11}$ The form in Eq. (2) was subsequently introduced in order to reduce the number of free parameters of the PES and to simplify its evaluation while retaining the essential shape (saddle and maximum energy, and curvature around the minimum). ${ }^{15}$

\section{CONCLUSIONS}

A systematic DFT investigation of the alkali-atom group adsorbed on $\mathrm{Cu}(001)$ has been performed to determine the lateral adiabatic potential-energy surface of individual adatoms. Convergence of results with respect to the calculations unit cell is accelerated by removing the dipole-dipole interaction among the adatom replicas. The monotonic variation in dipole moment, bond length, and PES corrugation from $\mathrm{Li}$ to Cs has been discussed. All atoms are found to adsorb in $H$ sites while $B$ is a saddle point and $T$ a PES maximum. The value of the saddle-point energy for $\mathrm{Li}(143 \mathrm{meV})$ is 1 order of magnitude larger than the one for Cs $(13 \mathrm{meV})$. Structural optimization induced by the adsorbate in the substrate can account up to $50 \%$ of the PES corrugation, even though the absolute displacement of substrate atoms is moderate. The energy cost of such substrate deformation is compatible with an harmonic response to the adsorbate. The DFT PES between high-symmetry configurations is very accurately described by a simple nonseparable cosine expression. Results are in good agreement with experimental characterizations, in particular, with the PES corrugation at the saddle point measured in He-atom scattering experiments. This agreement illustrates the accuracy of DFT calculations for this class of systems.

\section{ACKNOWLEDGMENTS}

This work was supported in part by the EU Network of Excellence NANOQUANTA (Grant No. NMP4-CT-2004500198) and the MIUR of Italy (Grant No. 2005021433003). Computational resources were also made available by CINECA through INFM grants. I am grateful to G. Alexandrowicz, M.I. Trioni, and G.P. Brivio for useful discussions.
${ }^{1}$ I. Langmuir, J. Am. Chem. Soc. 54, 2798 (1932).

${ }^{2}$ R. W. Gurney, Phys. Rev. 47, 479 (1935).

${ }^{3}$ H. Ishida, Phys. Rev. B 42, 10899 (1990).

${ }^{4}$ G. Pacchioni and P. S. Bagus, Surf. Sci. 269-270, 669 (1992).

${ }^{5}$ J. Bormet, J. Neugebauer, and M. Scheffler, Phys. Rev. B 49, 17242 (1994).

${ }^{6}$ R. D. Diehl and R. McGrath, J. Phys.: Condens. Matter 9, 951 (1997).

${ }^{7}$ J. Zhao, N. Pontius, A. Winkelmann, V. Sametoglu, A. Kubo, A. G. Borisov, D. Sánchez-Portal, V. M. Silkin, E. V. Chulkov, P.
M. Echenique, and H. Petek, Phys. Rev. B 78, 085419 (2008).

${ }^{8}$ A. P. Jardine, J. Ellis, and W. Allison, J. Chem. Phys. 120, 8724 (2004).

${ }^{9}$ R. Guantes, J. L. Vega, S. Miret-Artés, and E. Pollak, J. Chem. Phys. 119, 2780 (2003).

${ }^{10}$ G. Alexandrowicz, P. R. Kole, E. Y. M. Lee, H. Hedgeland, R. Ferrando, A. P. Jardine, W. Allison, and J. Ellis, J. Am. Chem. Soc. 130, 6789 (2008).

${ }^{11}$ A. P. Graham, F. Hofmann, J. P. Toennies, L. Y. Chen, and S. C. Ying, Phys. Rev. Lett. 78, 3900 (1997). 
${ }^{12}$ A. P. Jardine, S. Dworski, P. Fouquet, G. Alexandrowicz, D. J. Riley, G. Y. H. Lee, J. Ellis, and W. Allison, Science 304, 1790 (2004).

${ }^{13}$ G. Alexandrowicz, A. P. Jardine, H. Hedgeland, W. Allison, and J. Ellis, Phys. Rev. Lett. 97, 156103 (2006).

${ }^{14}$ A. Cucchetti and S. C. Ying, Phys. Rev. B 60, 11110 (1999).

15 J. Ellis, A. P. Graham, F. Hofmann, and J. P. Toennies, Phys. Rev. B 63, 195408 (2001).

${ }^{16} \mathrm{G}$. Fratesi, G. Alexandrowicz, M. I. Trioni, G. P. Brivio, and W. Allison, Phys. Rev. B 77, 235444 (2008).

${ }^{17}$ M. I. Trioni, G. Fratesi, S. Achilli, and G. P. Brivio, J. Phys.: Condens. Matter 21, 264003 (2009).

${ }^{18}$ N. D. Lang, Phys. Rev. B 4, 4234 (1971).

${ }^{19}$ J. Neugebauer and M. Scheffler, Phys. Rev. Lett. 71, 577 (1993).

${ }^{20}$ T. Oguchi and N. Hamada, J. Phys. Soc. Jpn. 66, 2751 (1997).

${ }^{21}$ G. P. Brivio, G. Butti, S. Caravati, G. Fratesi, and M. I. Trioni, J. Phys.: Condens. Matter 19, 305005 (2007).

${ }^{22}$ B. Sun, P. Zhang, S. Duan, X.-G. Zhao, and Q.-K. Xue, Phys. Rev. B 75, 245422 (2007).

${ }^{23}$ C. A. Papageorgopoulos, Phys. Rev. B 25, 3740 (1982).

${ }^{24}$ J. Cousty, R. Riwan, and P. Soukiassian, Surf. Sci. 152-153, 297 (1985).

${ }^{25}$ A. Ignaczak, J. Electroanal. Chem. 495, 160 (2001).

${ }^{26}$ W. Kohn and K. H. Lau, Solid State Commun. 18, 553 (1976).

${ }^{27}$ K. H. Lau and W. Kohn, Surf. Sci. 65, 607 (1977).

${ }^{28}$ K. H. Lau and W. Kohn, Surf. Sci. 75, 69 (1978).

${ }^{29}$ L. Bengtsson, Phys. Rev. B 59, 12301 (1999).

${ }^{30}$ J. P. Perdew, K. Burke, and M. Ernzerhof, Phys. Rev. Lett. 77, 3865 (1996).

${ }^{31}$ D. Vanderbilt, Phys. Rev. B 41, 7892 (1990).

${ }^{32}$ QUANTUM-ESPRESSO is a community project for high-quality quantum-simulation software, based on density-functional theory, and coordinated by Paolo Giannozzi. See http:// www.quantum-espresso.org and http://pwscf.org

${ }^{33}$ H. J. Monkhorst and J. D. Pack, Phys. Rev. B 13, 5188 (1976).
${ }^{34}$ C. Kittel, Introduction to Solid State Physics (Wiley, New York, 1986).

${ }^{35}$ S. Mizuno, H. Tochihara, and T. Kawamura, Phys. Rev. B 50, 17540 (1994).

${ }^{36}$ T. Aruga, H. Tochihara, and Y. Murata, Phys. Rev. B 34, 8237 (1986).

${ }^{37}$ J. W. Gadzuk, Phys. Rev. B 79, 073411 (2009).

${ }^{38}$ S. Mizuno, H. Tochihara, and T. Kawamura, Surf. Sci. 293, 239 (1993).

${ }^{39}$ A. Mikkelsen and D. L. Adams, Phys. Rev. B 60, 2040 (1999).

${ }^{40}$ H. L. Meyerheim, J. Wever, V. Jahns, W. Moritz, P. J. Eng, and I. K. Robinson, Surf. Sci. 304, 267 (1994).

${ }^{41}$ G. S.-M. Tong and A. S.-C. Cheung, J. Phys. Chem. A 106, 11637 (2002).

${ }^{42}$ R. D. Diehl and R. McGrath, Surf. Sci. Rep. 23, 43 (1996).

${ }^{43}$ S. Quassowski and K. Hermann, Surf. Rev. Lett. 4, 1209 (1997).

${ }^{44}$ R. D. Shannon, Acta Crystallogr., Sect. A: Cryst. Phys., Diffr., Theor. Gen. Crystallogr. 32, 751 (1976).

${ }^{45}$ S. Mizuno, H. Tochihara, and T. Kawamura, Surf. Sci. Lett. 292, L811 (1993).

${ }^{46}$ A. P. Jardine, G. Alexandrowicz, H. Hedgeland, R. D. Diehl, W. Allison, and J. Ellis, J. Phys.: Condens. Matter 19, 305010 (2007).

${ }^{47}$ H. Hedgeland, P. R. Kole, H. R. Davies, A. P. Jardine, G. Alexandrowicz, W. Allison, J. Ellis, G. Fratesi, and G. P. Brivio (unpublished).

${ }^{48}$ For simplicity of notation let us consider the energy of the surface as depending on only one coordinate, $u$ : $E_{\text {surf }}^{\mathrm{tot}}(u)=K u^{2} / 2$ (terms not depending on $u$ are omitted). In presence of the adatom, $E_{\mathrm{A} / \text { surf }}^{\mathrm{tot}}(u)=K u^{2} / 2-F u$, which is minimized for $u=\bar{u}$ $\equiv F / K$ (the coordinate after structural optimization), where it takes the value $-F^{2} / 2 K$. Not allowing relaxation would result in the energy $E_{\mathrm{A} / \text { surf-frozen }}^{\mathrm{tot}} \equiv E_{\mathrm{A} / \text { surf }}^{\mathrm{tot}}(0)=0$, hence, $E^{\text {relax }} \equiv E_{\mathrm{A} / \text { surf }}^{\mathrm{tot}}(0)$ $-E_{\mathrm{A} / \text { surf }}^{\text {tot }}(\bar{u})=F^{2} / 2 K$. Similarly, the energy of the deformed surface is $E_{\text {surf-def }}^{\text {tot }} \equiv E_{\text {surf }}^{\text {tot }}(\bar{u})=F^{2} / 2 K$, which results in $E^{\text {def }}$ $\equiv E_{\text {surf }}^{\mathrm{tot}}(\bar{u})-E_{\text {surf }}^{\mathrm{tot}}(0)=F^{2} / 2 K$. 